\title{
Sharing Video Browsing Style by Associating Browsing Behavior with Low-Level Features of Videos
}

\author{
Akio Takashima and Yuzuru Tanaka \\ Meme Media Laboratory, West8. North13, Kita-ku \\ Sapporo Hokkaido, Japan \\ \{akiota, tanaka\}@meme.hokudai.ac.jp
}

\begin{abstract}
This paper focuses on a method to extract video browsing styles and reusing it. In video browsing process for knowledge work, users often develop their own browsing styles to explore the videos because the domain knowledge of contents is not enough, and then the users interact with videos according to their browsing style. The User Experience Reproducer enables users to browse new videos according to their own browsing style or other users' browsing styles. The preliminary user studies show that video browsing styles can be reused to other videos.
\end{abstract}

Keywords: video browsing, active watching, tacit knowledge.

\section{Introduction}

The history of video browsing has been changing. We used to watch videos or TV programs passively (Fig. 1. (1)), then select videos on demand (Fig. 1. (2)). There are increasingly more opportunities to use video for knowledge work, such as monitoring events, reflecting on physical performances, or analyzing scientific experimental phenomena. In such ill-defined situations, users often develop their own browsing styles to explore the videos because the domain knowledge of contents is not useful, and then the users interact with videos according to their browsing style (Fig. 1. (3)) [1]. However, such kind of tacit knowledge, which is acquired through user's experiences [2], has not been well managed. The goal of our research is to share and reuse tacit knowledge in video browsing (Fig. 1. (4)). This paper focuses on a method to extract video browsing styles and reusing it.

To support video browsing process, numerous studies which focus on content based analysis for retrieving or summarizing video had been reported [3][4]. The content based knowledge may include semantic information of video data, in other words, generally accepted assumptions. For example, people tend to pay attention to goal scenes of soccer games, or captions on news program include the summary or location of the news topic, etc. Thus, this approaches only work on the specific purposes (e.g. extracting goal scenes of soccer games as important scenes) which are assumed beforehand. Several studies have been reported that address using users' behavior to estimate preferences of the users in web browsing process [5]. On the other hand, little is reported in video browsing process. 


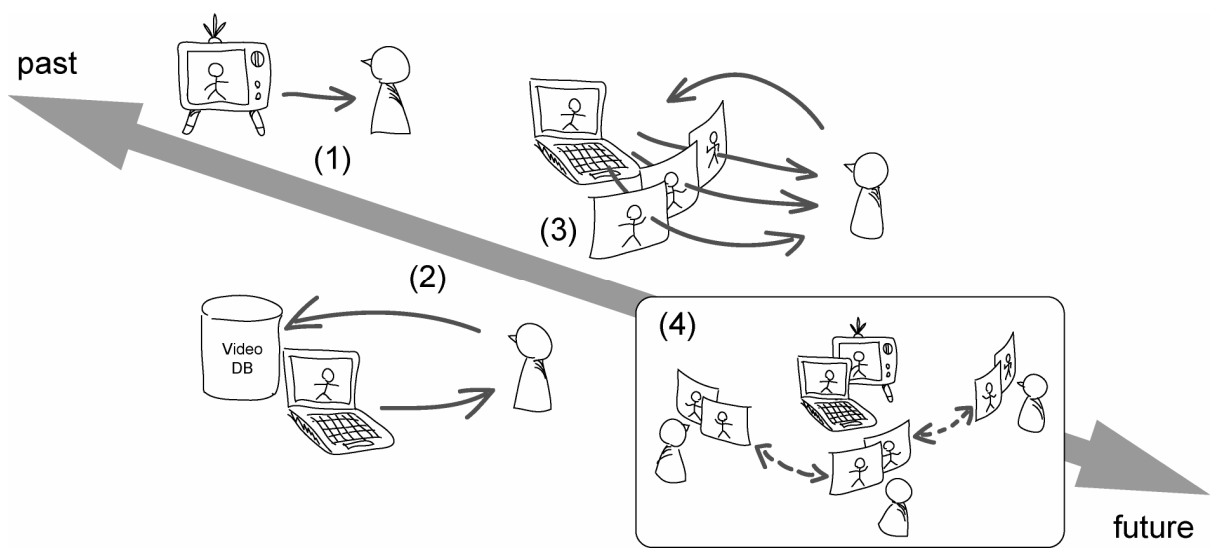

Fig. 1. The history of video browsing has been changing. We used to watch videos or TV programs passively (1), and then select videos on demand (2). Now we can interact with videos according to our own browsing style (3), however, we could not share these browsing styles. We assume that sharing them leads us to the next step of video browsing (4), especially in knowledge work.

In the area of knowledge management systems, many studies have been reported [6]. As media for editing and distributing and managing knowledge, Meme Media have been well known in the last decade [7]. However, target objects for reusing or sharing have been limited to the resources which are easily describable such as functions of software or services of web applications. In this work, we extend the area of this approach to more human side which treats indescribable resources such as know-how or skills of human behavior, in other words, tacit knowledge.

\section{Approach}

This paper focuses on a method to extract video browsing styles and reusing it. We assume the following characteristics in video browsing for knowledge work:

- People often browse video in consistent, specific manners

- User interaction with video can be associated with low-level features of the video

While user's manipulation to a video depends on the meanings of the content and on how the user's thought is, it is hard to observe these aspects. In this research, we tried to estimate associations between video features and user manipulations (Fig. 2). We treat the low-level features (e.g., color distribution, optical flow, and sound level) as what are associated with user manipulation. The user manipulation indicates changing speeds (e.g., Fast-forwarding, Rewinding, and Slow Playing). Identifying associations from these aspects, which can be easily observed, means that the user can grab tacit knowledge without domain knowledge of the content of the video. 


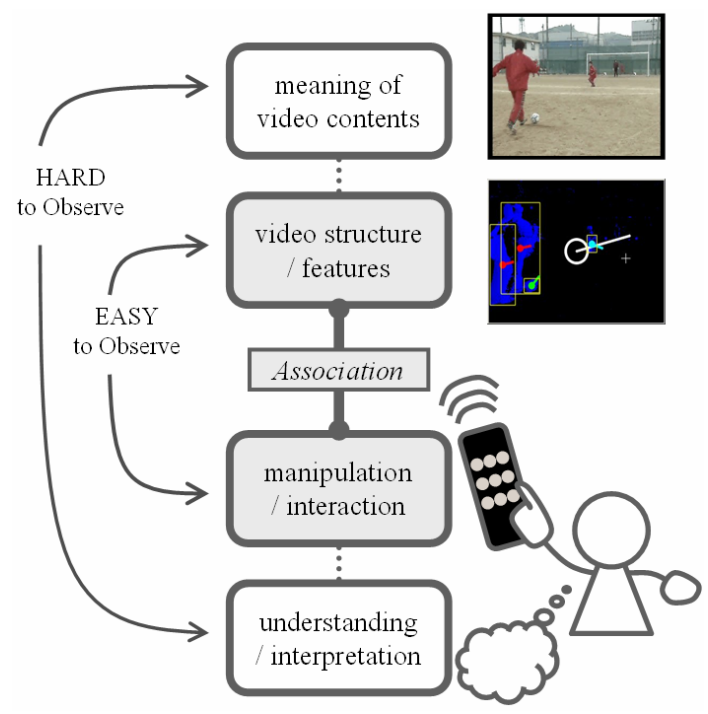

Fig. 2. While users' manipulations may depend on the meaning of the contents or users' understandings of the videos, it is difficult to observe these aspects. Therefore, we tried to estimate associations between easily observable aspects such as video features and user manipulations.

\section{The User Experience Reproducer}

\subsection{System Overview}

To extract associations between users' manipulations and low-level video features, and to reproduce browsing style for other videos, we have developed a system called the User Experience Reproducer.

The User Experience Reproducer consists of the Association Extractor and the Behavior Applier (Fig. 3).

The Association Extractor identifies relationships between low-level features of videos and user manipulation to the videos. The Association Extractor needs several training videos and the browsing logs by a particular user on these videos as input. To record the browsing logs, the user browses training videos using the simple video browser, which enables user to control playing speed. The browsing logs possess the pairs of a video frame number and the speed at which the user actually played the frame. As low-level features, the system analyzes more than sixty properties of each frame such as color dispersion, mean of color value, number of moving objects, optical flow, sound frequency, and so on. Then the browsing logs and the low-level features generate a classifier that determines the speed at which each frame of the videos should be played. In generating the classifier, we use WEKA engine that is a data mining software [8]. 


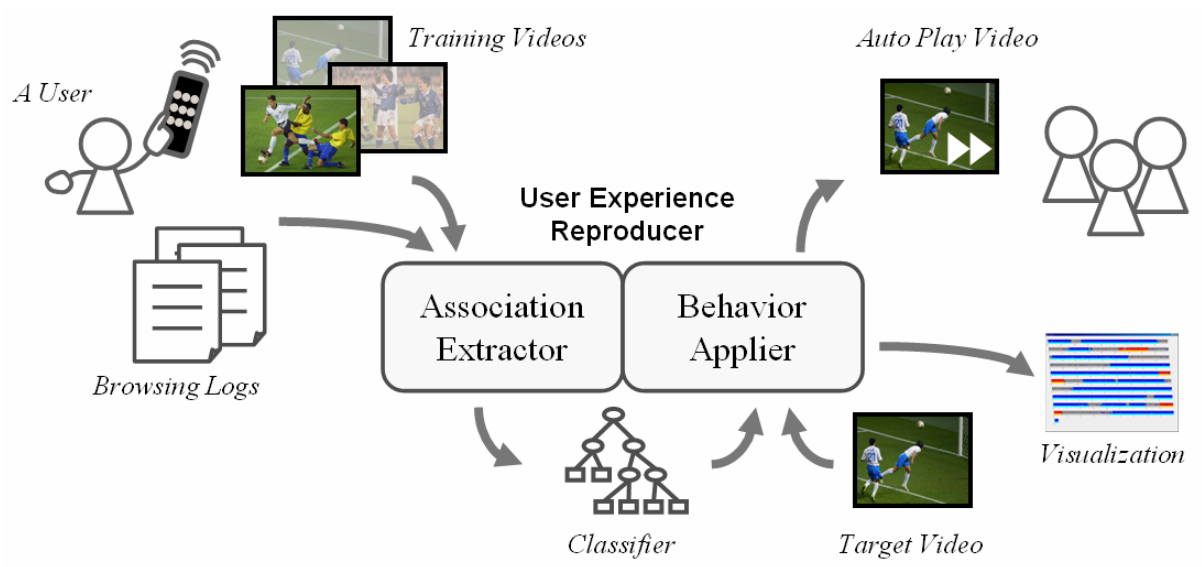

Fig. 3. The User Experience Reproducer consists of the Association Extractor and the Behavior Applier. The Association Extractor calculates associations using browsing logs and the lowlevel video features, and then creates a classifier. The Behavior Applier plays target video automatically based on the classification with the classifier.

The Behavior Applier plays the frames of a target video automatically at each speed in accordance with the classifier. The Behavior Applier can remove outliers from the sequence of frames, which should be played at the same speed, and also can visualize whole applied behavior to each frame of the video.

\subsection{The Association Extractor}

The Association Extractor identifies relationships between low-level features of video and user manipulation to the videos then generates a classifier. In this section we describe more details about the low-level features of video and user manipulations which are currently considered in the Association Extractor.

\section{Low-level features of video}

Video data possesses a lot of low-level features. Currently, the system can treat more than sixty features. These features are categorized into five aspects as follows:

- Statistical data of color values in a frame

- Representative color data

- Optical flow data

- Number of moving objects

- Sound levels

\section{Statistical data of color values in a frame}

As a most simple low-level feature, we treat the statistical data of color values in each frame of a video, for example, the mean and the standard deviation of Hue, Saturation, and Value (Brightness). 


\section{Representative color data}

The system uses statistical data of the pixels which are painted a representative color. The representative color is a particular color space set beforehand (e.g. $30<\mathrm{H}<=50$, $80<\mathrm{S}<=90,75<\mathrm{V}<=95$ ). The system counts how many representative color pixels exist in a frame and calculates the mean of $\mathrm{X}$, Y position and their standard deviations (Fig. 4 (a)). Currently, the color space is specified manually, it can be specified automatically by calculating which colors are most frequently used through the entire video.

\section{Optical flow data}

As dynamic data of video, the system calculates optical flow of moving objects in a video. First, the system detects distinguishable pixels such as contours of human body or the corners of a goal post in soccer games. Then the system calculates the vector data of optical flow at each point, and calculates the mean angle and the magnitude of vector values in the twelve rectangular sub areas respectively (Fig. 4 (b)). These values may represent not only states of moving objects but also camera motions.

The number of the distinguishable pixels is also one of the features.

\section{Number of moving objects}

The system can detect moving object and then counts the number of objects according to its size (Fig. 4 (c)). If the video consistently records the same objects, each detection

(a) representative color data

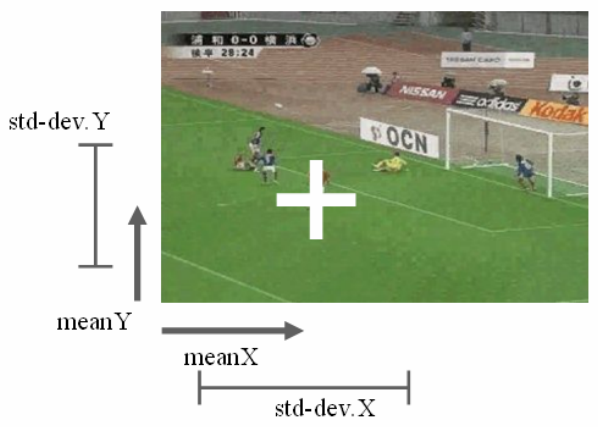

(b) optical flow data

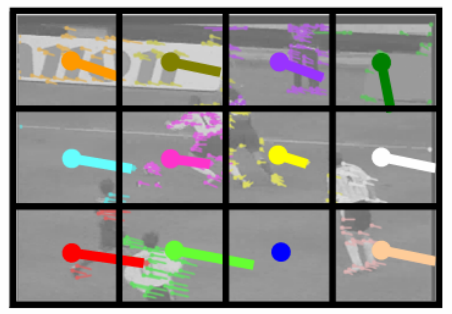

(c) number of moving objects

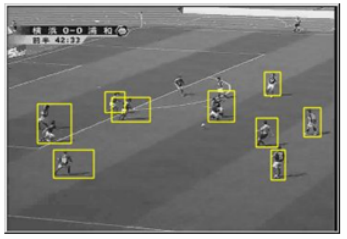

small

$(\sim 1 / 128$ of screen size $)$

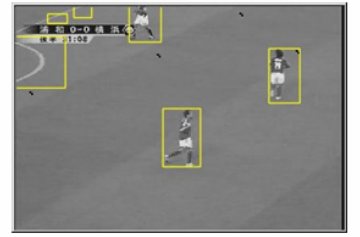

middle

$(1 / 128 \sim 1 / 8)$

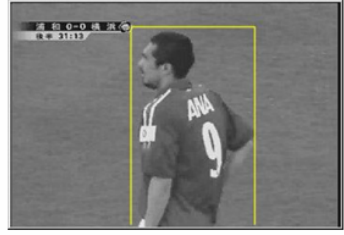

large

$(1 / 8 \sim)$

Fig. 4. The system makes associations between users' browsing speeds and low-level video features such as representative color data (a), optical flow data (b), and number of moving objects (c) 
of a small object means that it may have been recorded in a wide angle and each detection of a large object means the opposite case.

\section{Sound levels}

Sound levels are divided into ten groups based on its frequency (e.g. 0-32 Hz, 32$64 \mathrm{~Hz}, \ldots, 8000-10000 \mathrm{~Hz})$ and used as low-level features. If the video includes sound data, it can be one of good features of the video.

\section{User manipulations}

To record the browsing logs, the user browses training videos using the simple video browser, which enables user to control playing speed. We categorized the patterns of changing playing speeds into three types based on the patterns frequently used in informal user observation [9]. The three types are as follows:

- Skip

- Re-Examine

- Others

\section{Skip}

We regard the browsing with the speed higher than the normal playing speed (1.0x) as a skipping behavior.

\section{Re-Examine}

In exploring video, a user could re-check and focus on a frame that has just passed during browsing. When the pattern is made from forwarding at less than normal speed after the rewinding, we regard it as a re-examine behavior

\section{Others}

The speeds which are not described above are categorized into this class.

To avoid any conflict, we set the priority in the order of Re-Examine, Skip, and then Others.

\subsection{The Behavior Applier}

The Behavior Applier plays the frames of a target video automatically at each speed in accordance with the classifier.

\section{Mapping from User Manipulation into Auto Play Speed}

The user manipulations are categorized into three types described above, and the classifier tries to associate each video frame of a target video into these three types.

We designed the mapping from three types of behaviors into specific speeds (Fig. 5). The skip behavior is reproduced as faster playing (5.0x). The others are reproduced as playing at a normal speed. The re-examine behavior is reproduced as slower playing $(0.5 \mathrm{x})$. 


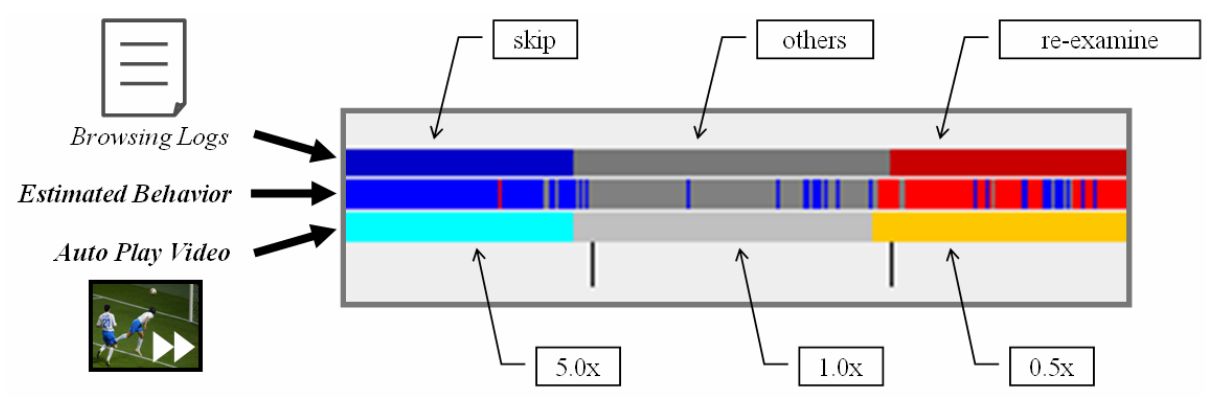

Fig. 5. This visualization shows that how target video will play at a various speeds. The three belts are indicates video data, in other words, accumulated video frames from left to right. The first belt indicates a browsing log of a video which used for training the classifier. The second belt shows estimated behaviors for a target video. In this figure, the same video is used as the one of training video and the target video for confirmation. Therefore the first and the second belt should be ideally the same. The third belt indicates the noise reduced version of the second belt. The target video will play at each speed written in the bottom of this figure.

\section{User Study}

We conducted a preliminary user study to extract and reuse one's browsing style.

\section{Setting}

In this study, we used ten $5 \mathrm{~min}$. soccer game videos for training a classifier, and two $5 \mathrm{~min}$. soccer game videos for applying the browsing style and for playing automatically. The number of recorded soccer games is three and the training videos are some fragments of these games. The target videos are not included in training videos. We observed two subjects so that the process described above was conducted twice. Each subject is a normal computer user and asked to explore the training videos to find interest scenes of soccer games. After the system generates the classifiers for each of the users, the users watch two target videos playing automatically through each classifier, and then asked their impression.

\section{Overview of the results}

In training phase, subjectA tried to re-examine (rewind then play at less than normal speed) particular scenes, which show players gathering in front of goal post or show a player kicking the ball to the goal. In addition, he skipped out-of-play scenes and scenes do not display goal post. SubjectB tended to skip out-of-play scenes of the games.

Through the Behavior Applier, each subject saw the target videos playing automatically in accordance with their own browsing style. The trial of subjectA played nearly $80 \%$ of important (for subjectA) scenes at a slower speed. The trial of SubjectB skipped nearly $70 \%$ of out-of-play scenes. These percentages were calculated by measuring duration of these scenes manually. The results of informal interview tell that both subjects got satisfaction from the target videos, which are automatically played. 


\section{Discussion}

\subsection{Results of the User Study}

In our user study, we tried to quantize how many scenes which are meaningful for a user are detected. Although, it is not easy to describe whether the applied browsing behavior by the system constitutes a perfect fit for the user's particular needs, it seems possible to reuse tacit knowledge in video browsing without any domain knowledge of the contents.

The results of the user study might be too good. We think there are two reasons. First, the data which are used for training a classifier and playing target video are not sufficiently large. Although these videos are not the same, the videos are recorded by one TV station, so that the conditions of low-level features might not be so different. Secondly, the browsing styles were very consistent because the two subjects both had their own browsing style at least for soccer games. If the subjects have not browsed video so consistently, the result might be worse.

\section{Kind of attribute for association}

In this work, the Association Extractor considers more than sixty features to generate a classifier. Video data has more and more features that we can observe, and we think that the result will be better if the system can treat more features.

On the other hand, we categorized users' manipulations into some meaningful patterns such as skipping and re-examining then we use only three categories to be associated with a lot of low-level features. It is because that the system may not generate any associations if both sides (user behavior and video features) have a lot of attributes. Estimating several patterns of browsing behavior [10] is one of our future work to regard users' manipulations as low-level actions.

\section{Conclusion}

How people interact with text and images in everyday life involves not only simple naive information-receiving processes but also complex knowledge-construction processes. Videos as knowledge materials are no exception. In video browsing especially for knowledge work, sharing and reusing know-how of video browsing plays crucial role in solving problems. In this paper, we described a way of reusing such knowledge without any domain knowledge of videos.

We plan to conduct additional experiments and to develop a framework that allows users to combine several video browsing styles to obtain a composite style.

\section{References}

1. Yamamoto, Y., Nakakoji, K., Takashima, A.: The Landscape of Time-based Visual Presentation Primitives for Richer Video Experience. In: Costabile, M.F., Paternó, F. (eds.) INTERACT 2005. LNCS, vol. 3585, pp. 795-808. Springer, Heidelberg (2005)

2. Polanyi, M.: Tacit Dimension, Peter Smith Pub Inc. (1983) 
3. Nakamura, Y., Kanade, T.: Semantic analysis for video contents extraction-spotting by association in news video. In: Proceedings of the fifth ACM international conference on Multimedia, Seattle, pp. 393-401 (1997)

4. Ekin, A., Tekalp, A.M., Mehrotra, R.: Automatic soccer video analysis and summarization. IEEE Trans. on Image Processing 12(7), 796-807 (2003)

5. Seo, Y., Zhang, B.: Learning user's preferences by analyzing web-browsing behaviors. In: Proceedings of International Conference on Autonomous Agents, pp. 381-387 (2000)

6. Alavi, M., Leidner, D.E.: Knowledge management systems: issues, challenges, and benefits. Journal of Commun. AIS, AIS, Atlanta, GA, 1(2) (1999)

7. Tanaka, Y.: Meme Media and Meme Market Architectures: Knowledge Media for Editing, Distributing, and Managing Intellectual Resources. IEEE Press, New York (2003)

8. WEKA: http://www.cs.waikato.ac.nz/ml/weka/

9. Takashima, A., Yamamoto, Y., Nakakoji, K.: A Model a Tool for Active Watching: Knowledge Construction through Interacting with Video. In: Proceedings of INTERACTION: Systems, Practice and Theory, pp. 331-358 (2004)

10. Syeda-Mahmood, T., Ponceleon, D.: Learning video browsing behavior and its application in the generation of video previews. In: Proceedings of the ninth ACM international conference on Multimedia, pp. 119-128 (2001) 